\title{
Survey of Penicillia associated with Italian grana cheese
}

\author{
S. Decontardi ${ }^{\text {a }}$, A. Mauro ${ }^{\text {a }}$, N. Lima ${ }^{\text {b }}$, P. Battilani ${ }^{\text {a,* }}$ \\ a Department of Sustainable Crop Production, Università Cattolica del Sacro Cuore, Via Emilia Parmense 84, 29122 Piacenza, Italy \\ b CEB-Center of Biological Engineering, Micoteca da Universidade do Minho, 4704-553 Braga, Portugal
}

\section{A R T I C L E I N F O}

\section{Article history:}

Received 14 October 2016

Received in revised form 18 January 2017

Accepted 30 January 2017

Available online 31 January 2017

\section{Keywords:}

Cheese

Citrinin

Fungal identification

Ochratoxin A

Penicillium

\begin{abstract}
A B S T R A C T
The present work aimed to contribute information on the mycobiota associated with ripening grana cheese, with focus on the genus Penicillium as potential mycotoxin producers. Eighteen wheels of grana cheese, aged in different storehouses situated in Northern Italy, were sampled to isolate associated fungi. Penicillium spp. were commonly dominant; morphological observation and gene sequencing were applied to identify Penicillium at species level. P. crustosum and P. solitum were the dominant species. Citrinin and ochratoxin A mycotoxins were analysed and the latter was found in all grana cheese samples. These results confirmed that a polyphasic approach is mandatory for Penicillium identification at species level.
\end{abstract}

(C) 2017 Elsevier B.V. All rights reserved.

\section{Introduction}

Grana is an Italian hard cheese, made from unpasteurised, partially skimmed cow's milk, aged for a period ranging from nine months up to four years. The word "grana" comes from "grainy" to describe its fine granular texture. This cheese is of utmost importance in Italian food culture and economy. The Protected Designation of Origin (PDO) is attributed to "Grana Padano" and "Parmigiano Reggiano" brands. Nevertheless, information on the mycobiota associated with this type of cheese is poor, with only one paper published on this topic in recent decades (Dragoni et al., 1983). The development of uncontrolled moulds during ripening and ageing has been reported for other cheese types, causing spoilage and possibly leading to mycotoxin production (Ropars et al., 2012). The genera Aspergillus, Cladosporium, Geotrichum, Mucor, Penicillium, Trichoderma (Sengun et al., 2008) and Fusarium (Lund et al., 1995; Montagna et al., 2004) have been reported as cheese contaminants. In addition, some related mycotoxins such as citrinin (CIT) (Bailly et al., 2002; Cooper et al., 1982; Ostry et al., 2013; Pugazhenthi et al., 2000) and ochratoxin A (OTA) (Dall'Asta et al., 2008; Jarvis, 1983) have been detected in cheese. In particular, OTA contamination was recently reported in packed grated cheese, commonly produced with grana cheese (Biancardi et al., 2013). Mycotoxins are a main concern in food and feed: CIT showed nephrotoxic, immunosuppresive, teratogenic and mutagenic effects in past studies, and seems able to cause hemolysis of human erythrocytes (Ambrose and Deeds, 1946; Houbraken et al., 2010; IARC, 1986; Lurá et al., 2004; Ostry et al., 2013) while OTA is mainly toxic for the kidneys and liver

\footnotetext{
* Corresponding author.

E-mail address: paola.battilani@unicatt.it (P. Battilani).
}

(Richard, 2007) and classified in group 2B by the International Agency for Research on Cancer (IARC, 1987). Therefore, the present work aimed to contribute by filling in these gaps in knowledge with a first description of the mycobiota associated with ripening grana cheese, with focus on the genus Penicillium, as potential mycotoxin producers. A polyphasic approach, based on morphological and molecular observations and on mycotoxin analysis, was used.

\section{Materials and methods}

\subsection{Chemicals}

Mycological peptone, microbiological grade agar, malt extract and yeast extract were purchased from Himedia Laboratories (Mumbai, India); Dichloran Rose Bengal Chloramphenicol Agar (DRBC) and Czapek Dox Agar (CZ) ready prepared culture media were purchased from Oxoid (Basingstoke, U.K.); $\mathrm{D}(+)$ glucose was purchased from Sigma-Aldrich (Merck, Darmstadt, Germany); salts $\left(\mathrm{CuSO}_{4} \cdot 5 \mathrm{H}_{2} \mathrm{O}\right.$; $\mathrm{ZnSO}_{4} \cdot 7 \mathrm{H}_{2} \mathrm{O} ; \mathrm{K}_{2} \mathrm{HPO}_{4} \cdot 3 \mathrm{H}_{2} \mathrm{O} ; \mathrm{MgSO}_{4} \cdot 7 \mathrm{H}_{2} \mathrm{O} ; \mathrm{KCl} ; \mathrm{FeSO}_{4} \cdot 7 \mathrm{H}_{2} \mathrm{O}$ ) were purchased form Carlo Erba Reagents S. r. l. (Milano, Italy); a DNA "Plant II" extraction kit was purchased from Macherey-Nagel (Düren, Germany); PCR master mix was purchased from Promega Corporation (Madison, Wisconsin, USA); an Exosap cleaning kit was purchased from Euroclone (Pero (MI), Italy).

\subsection{Sampling}

Eighteen ripening storehouses for grana cheese were sampled in 2013, in five sites in northern Italy. Seven storehouses (1-7) were 
located in Brescia (BS), four in Cremona (8-11, CR), four in Mantova $(12-15, \mathrm{MN})$, two in Piacenza (16 and 17, PC) and one in Verona (18, VR).

One ripening wheel was randomly chosen for each storehouse and sampled collecting $10 \mathrm{~g}$ of cheese rind, scratched from 5 different areas, to $0.5 \mathrm{~mm}$ depth.

\subsection{Mycological analysis}

One gram of scratched cheese rind was sub-sampled and diluted in $9 \mathrm{~mL}$ of physiological solution (1\% peptone), accurately mixed and serially diluted up to $10^{-7}$. Dilutions were plated in triplicate using DRBC agar medium and incubated for 7 days in the dark at $25^{\circ} \mathrm{C}$. Growing colonies were counted and reported as colony forming units per $\mathrm{g}$ of cheese rind $(\mathrm{CFU} / \mathrm{g})$ as a rough estimation of fungal bioload. Fungi were also identified to genus level using optical microscopy.

\subsubsection{Strain selection}

Three to five representative colonies of Penicillium were selected from each wheel, repeatedly transferred onto $\mathrm{CZ}$ to obtain pure cultures and subsequently managed to obtain monosporic strains destined for identification at species level.

Monosporic cultures were grown on Blakeslee Malt Extract Autolysate Agar (MEA, Samson and Frisvad, 2004; malt extract 30 g; mycological peptone $1 \mathrm{~g}$; glucose $20 \mathrm{~g}$; $\mathrm{CuSO}_{4} \cdot 5 \mathrm{H}_{2} \mathrm{O} 0.005 \mathrm{~g} ; \mathrm{ZnSO}_{4} \cdot 7 \mathrm{H}_{2} \mathrm{O}$ $0.01 \mathrm{~g}$; agar $20 \mathrm{~g}$; bidistilled water $1 \mathrm{~L}$, adjusted to $\mathrm{pH} 5.3 \pm 0.3$ ), at $25^{\circ} \mathrm{C}$ in the dark for one week. Plugs were then removed from each colony, put into bidistilled sterile water and stored at $4{ }^{\circ} \mathrm{C}$ until their use.

\subsubsection{Morphological identification}

All monosporic strains were 3-point inoculated on Czapek Yeast Agar (CYA, Samson and Frisvad, 2004; yeast extract $5 \mathrm{~g}$; sucrose $30 \mathrm{~g}$; $\mathrm{K}_{2} \mathrm{HPO}_{4} \cdot 3 \mathrm{H}_{2} \mathrm{O} 1.3 \mathrm{~g} ; \mathrm{MgSO}_{4} \cdot 7 \mathrm{H}_{2} \mathrm{O} 0.5 \mathrm{~g} ; \mathrm{KCl} 0.5 \mathrm{~g} ; \mathrm{FeSO}_{4} \cdot 7 \mathrm{H}_{2} \mathrm{O}$ $0.01 \mathrm{~g} ; \mathrm{CuSO}_{4} \cdot 5 \mathrm{H}_{2} \mathrm{O} 0.005 \mathrm{~g} ; \mathrm{ZnSO}_{4} \cdot 7 \mathrm{H}_{2} \mathrm{O} 0.01 \mathrm{~g}$; agar $15 \mathrm{~g}$; bidistilled water $1 \mathrm{~L}$, adjusted to $\mathrm{pH} 6.3 \pm 0.2$ ) and MEA and incubated at $25^{\circ} \mathrm{C}$ in the dark for 7 days. At the end of incubation, they were observed for macroscopic (colony diameter, obverse and reverse colour, margins, presence/absence of exudate droplets) and microscopic characters ( shape and size of penicillus and spores, roughness of the stipe) and tentatively grouped at species level.

Reference strains of the potential species of interest were obtained from culture collections (Table 1) and used as reference in the following steps of the study for comparisons with the strains selected according to Section 2.3.1.

\subsubsection{Molecular identification}

Monosporic strains were identified by BenA (encoding for $\beta$-Tubulin) and $\mathrm{COI}$ (encoding for Cytochrome Oxidase subunit I) gene sequencing. Samples were inoculated in $8 \mathrm{~mL}$ of Malt Extract Broth (MEB, Pitt, 1979; malt extract $30 \mathrm{~g}$; mycological peptone $1 \mathrm{~g}$; glucose $20 \mathrm{~g}$; bidistilled water $1 \mathrm{~L}$ ) and incubated at room temperature for

\section{Table 1}

Reference strains from fungal collections used in this work.

\begin{tabular}{llll}
\hline Fungal collection & Strain code & Origin & Species \\
\hline CBS & 115992 & The Netherlands & P. citrinum \\
CBS & 122396 & USA & P. citrinum \\
CBS & 101025 & Portugal & P. crustosum \\
CBS & 115503 & Scotland & P. crustosum \\
BFE & 856 & Italy & P. nordicum \\
CBS & 110769 & Spain & P. nordicum \\
CBS & 112573 & Italy & P. nordicum \\
CBS & 323.92 & Denmark & P. verrucosum \\
CBS & 325.92 & Denmark & P. verrucosum \\
\hline
\end{tabular}

CBS: CBS-KNAW Fungal Biodiversity Centre, The Netherlands.

BFE: Federal Research Centre for Nutrition and Food, Germany.
7 days in $15 \mathrm{~mL}$ Falcon ${ }^{\circledR}$ tubes; the tubes were kept on moving using an orbital shaker $(100 \mathrm{rpm})$ for the whole incubation period.

DNA was extracted using a "Plant II" kit according to the handbook supplied by the producer. Diluted genomic DNA (1:10, about $2 \mathrm{ng} / \mu \mathrm{L})$ was employed for PCR amplification using the primer pairs $B t 2 a-B t 2 b$ (Glass and Donaldson, 1995) for BenA and PenF1-AspR1 for COI (Seifert et al., 2007).

PCR reactions for both genes were performed in $7 \mu \mathrm{L}$ reaction mixtures containing $3.5 \mu \mathrm{L}$ Promega master mix (Promega Corporation, Wisconsin, USA), $1.5 \mu \mathrm{L}$ genomic DNA, $0.25 \mu \mathrm{L}$ of each primer $(0.5 \mu \mathrm{M})$ and $1.5 \mu \mathrm{L}$ bidistilled sterile water. Amplification conditions were the following: $95{ }^{\circ} \mathrm{C}$ for $2 \mathrm{~min}, 35$ cycles at $95{ }^{\circ} \mathrm{C}$ for $1 \mathrm{~min}, 55^{\circ} \mathrm{C}$ for $45 \mathrm{~s}$, and $72{ }^{\circ} \mathrm{C}$ for $1 \mathrm{~min}, 72^{\circ} \mathrm{C}$ for $10 \mathrm{~min}$ and rest at $4{ }^{\circ} \mathrm{C}$.

Cleaning of the PCR product was performed using an Exosap kit according to the protocol provided by the producer. Sequencing was performed using a 48-capillary 3730 DNA Analyser (Thermo Fisher Scientific Inc., Waltham, MA, USA).

Sequences (forward and reverse) provided in AB1 format file were opened using the pattern analysis software package Bioedit Sequence Alignment Editor v.7.2.5.0 (Alzohairy, 2011) and processed; identification at the species level was performed using BLAST (https://blast. ncbi.nlm.nih.gov/Blast.cgi). Dendrograms were then deduced opening the final sequence using the pattern analysis software package Mega7 (Molecular Evolutionary Genetics Analysis version 7.0 for bigger datasets; Kumar et al., 2016) and using the Neighbor-Joining (NJ) method for dendrogram design (Saitou and Nei, 1987). The robustness of tree topology for each analysis was evaluated by 1000 bootstrap replicates in both cases.

Sequences of reference strains coming from official culture collections were obtained from the website of the respective collection, from the GenBank database (http://www.ncbi.nlm.nih.gov/genbank/), or by sequencing according to Section 2.3.3 if not available, and included in the dendrograms for clustering. More sequences were obtained from the GenBank database in case unexpected species were detected (type or neotype strains were chosen when possible).

\subsection{Mycotoxin analysis}

CIT and OTA were analysed in the 18 cheese samples, according to Somorin et al. (2016), and reported in $\mu \mathrm{g} / \mathrm{kg}$. Briefly, $9 \mathrm{~g}$ of scratched cheese rind were used; CIT was extracted using $50 \mathrm{~mL}$ of ortho-phosphoric acid and $10 \mathrm{mM}$ methanol solution $(3: 7 \mathrm{v} / \mathrm{v})$ using a rotary-shaking stirrer; then, after filtration through a folded filter paper, $2 \mathrm{~mL}$ of the filtrate were diluted with $20 \mathrm{~mL}$ of PBS and purified through immunoaffinity columns (R-Biopharm, Darmstadt, Germany). The column was washed with $2 \mathrm{~mL}$ of PBS and CIT slowly eluted $(0.5 \mathrm{~mL} / \mathrm{min})$ with $4 \mathrm{~mL}$ of methanol into a graduated glass vial; the eluate was then concentrated under a gentle stream of nitrogen, brought to $1 \mathrm{~mL}$ with methanol and water solution $(3: 7 \mathrm{v} / \mathrm{v})$ and vortexed for a few seconds. The extract was filtered before LC-MS/MS analysis. The instrumental analysis was carried out using a LC-MS/MS system in positive mode consisting of a LC 1.4 Surveyor pump, a Quantum Discovery Max triple-quadrupole mass spectrometer (Thermo-Fisher Scientific, San Jose, California, USA) and a PAL 1.3.1 sampling system (CTC Analitycs AG, Zwingen, Switzerland). Excalibur 1.4 software (Thermo-Fisher Scientific, San Jose, CA, USA) controlled the whole system. Limits of detection (LOD) and of quantification (LOQ) were 0.5 and $1.5 \mu \mathrm{g} / \mathrm{kg}$, respectively.

OTA was quantified using a HPLC-FLD (fluorescence detector) system Perkin Elmer 200 (Perkin Elmer, Norwalk, Connecticut, USA) after extraction with $100 \mathrm{~mL}$ of sodium bicarbonate $0.13 \mathrm{M}$ methanol $(1: 1 \mathrm{v} / \mathrm{v})$ using a stirrer. The suspension was then filtered and $5 \mathrm{~mL}$ of the filtrate were diluted with PBS $(50 \mathrm{~mL})$ and purified through immunoaffinity columns (Ochratest WB, Vicam, Watertown, MA, USA), which was washed with $2 \mathrm{~mL}$ PBS so that OTA was slowly eluted $(0.5 \mathrm{~mL} / \mathrm{min})$ with $3 \mathrm{~mL}$ of acetonitrile into a glass vial. The eluate was then concentrated under a gentle stream of nitrogen, brought to $1 \mathrm{~mL}$ with 
acetonitrile and $2 \%$ acetic acid solution (41:59 v/v) and vortexed for some seconds. The extract was filtered before analysis. The HPLC Perkin Elmer 200 system (Perkin Elmer, Norwalk, CT, USA) was equipped with a Jasco AS 1555 sampling system and a FP 1520 fluorescence detector (Jasco Corporation, Tokyo, Japan). A Borwin 1.5 software (Jasco Corporation, Tokyo, Japan) allowed the whole system to work. LOD and LOQ were 0.1 and $0.3 \mu \mathrm{g} / \mathrm{kg}$, respectively.

\section{Results}

\subsection{Fungal bioload}

The eighteen cheese wheels sampled were all colonized by fungi varying between $10^{3}$ and $10^{7} \mathrm{CFU} / \mathrm{g}$, with Penicillium ranging from $10^{2}$ to $10^{6} \mathrm{CFU} / \mathrm{g}$. Penicillium was generally dominant with the exception

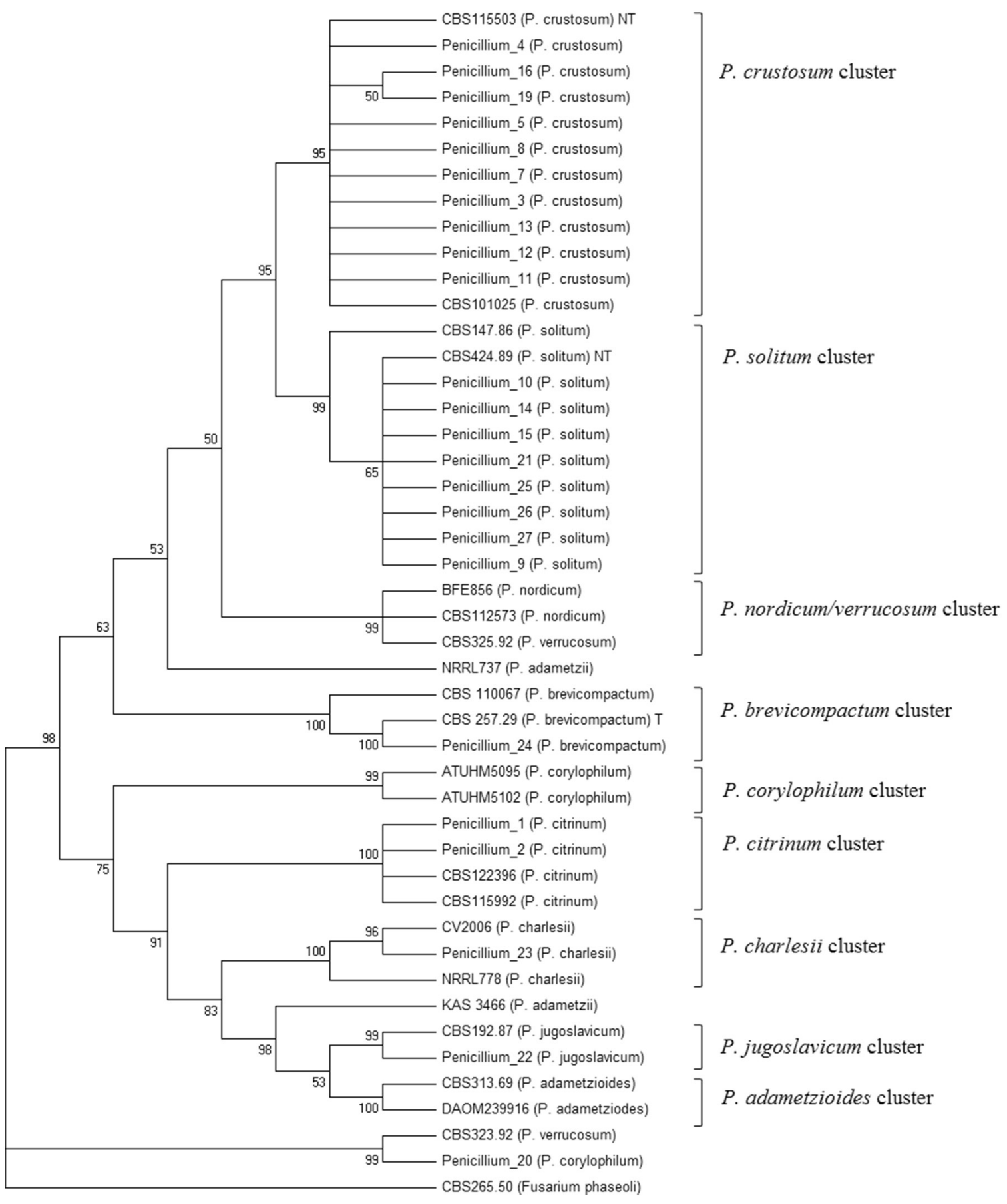

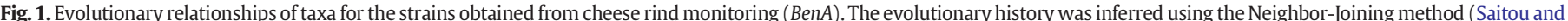

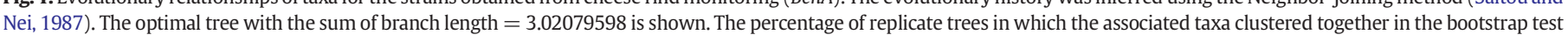

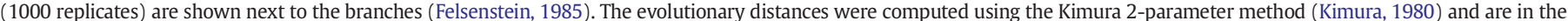

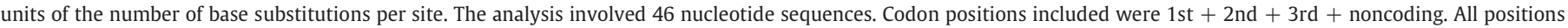

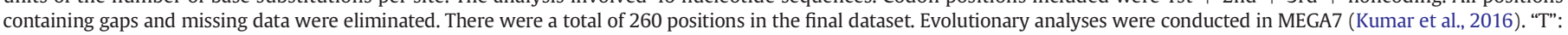
type strain. "NT": neotype strain. 
of samples 15, 17 and 18 where it was not isolated. Aspergilli were occasionally detected: Aspergillus section Flavi was isolated in samples coming from storehouses 10, 11 and 12. Cladosporium, Rhizopus and Trichoderma were also isolated.

\subsection{Morphological identification}

Twenty seven Penicillium spp. strains were selected in the survey; morphological observations of monosporic colonies grown on CYA

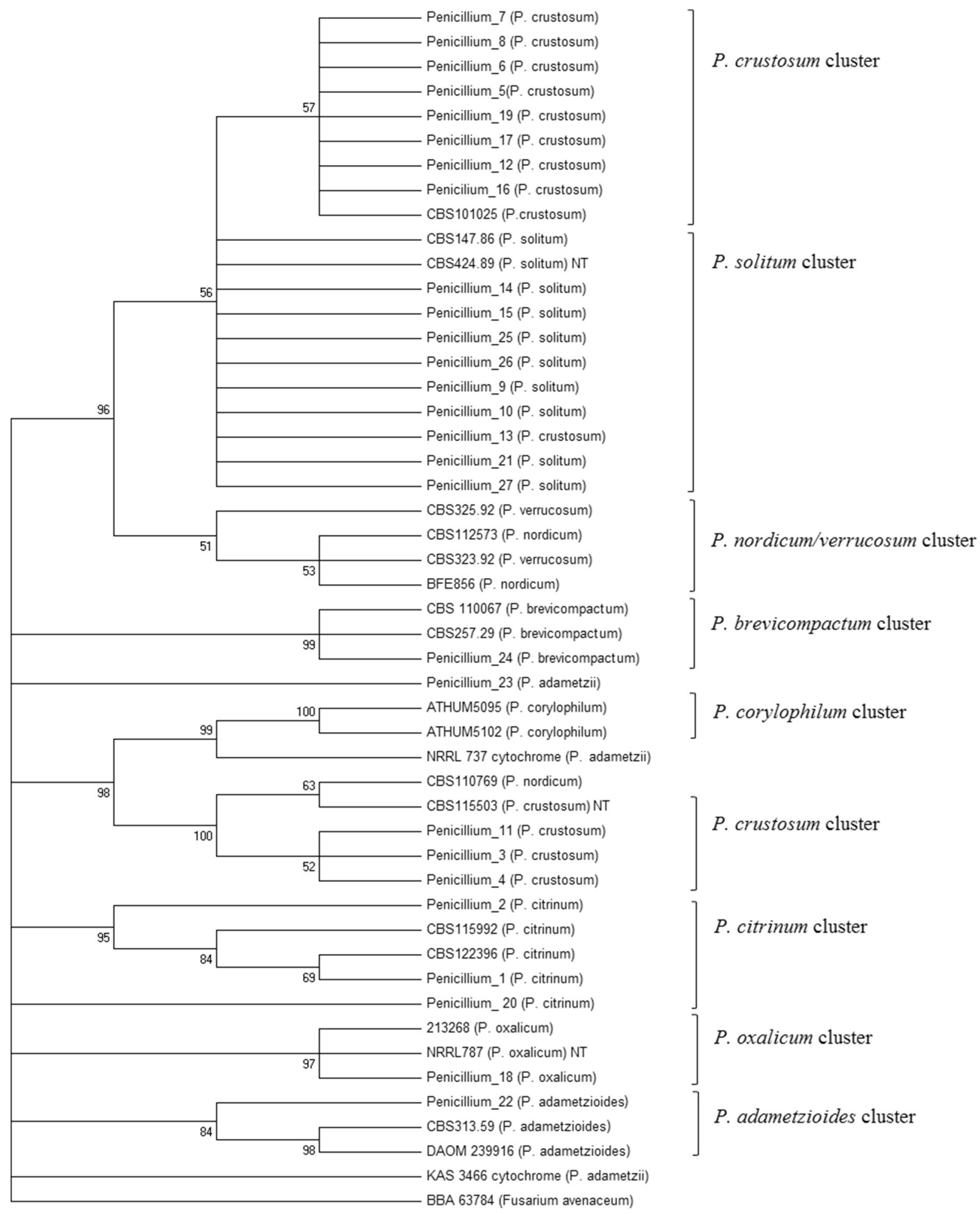

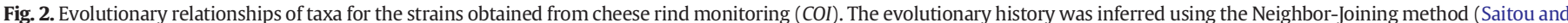

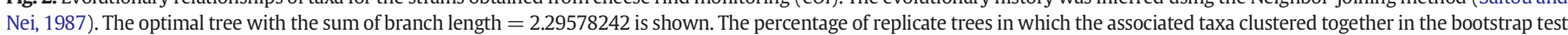

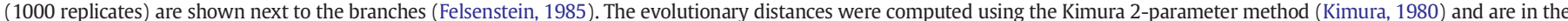

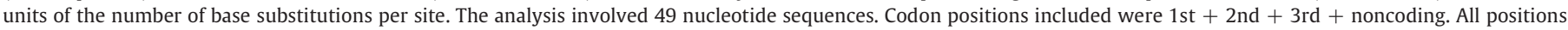

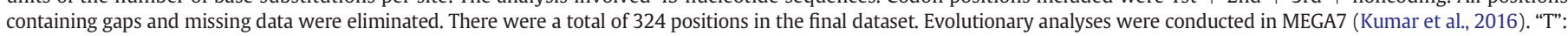
type strain. "NT": neotype strain. 
and MEA and visual comparison with reference strains led to the identification of the majority of them as $P$. crustosum, $P$. nalgiovense and $P$. citrinum, one as $P$. brevicompactum while 3 were not identified at the species level using this approach.

\subsection{Molecular identification}

Gene sequencing results (Table 3 ) were generally well supported by their respective dendrograms. In particular, for BenA (Fig. 1), all $P$. crustosum sequences available (10) clustered very well with their references, and the same occurred also for $P$. solitum (8 sequences), $P$. citrinum (2 sequences), P. brevicompactum, P. corylophilum, P. charlesii and $P$. jugoslavicum (1 sequence each). Those clusters were generally supported by high (>70\%) bootstrap values. Two outliers were found: the reference strain $P$. verrucosum CBS 323.92, not matching with the other $P$. nordicum/verrucosum reference strains and strain $n^{\circ} 20$ identified as $P$. corylophilum in BenA sequencing. Considering COI, sequencing results coming from BLAST analysis differed from BenA only in a few cases (samples 20, 22 and 23), with a poorer clustering (Fig. 2). Penicillium crustosum sequences were shared in two clusters: one at the top of the dendrogram, with 9 sequences of strains collected in the study and 1 reference, and the other in the middle, with 3 sequences from this study; one reference and sample $\mathrm{n}^{\circ} 13$ were outlier. Penicillium solitum sequences were shared in one cluster, with 8 sequences of strains collected in this study and 2 references, and 2 outliers. Considering the other species, the clustering was comparably good as for BenA, except for strain $n^{\circ} 20$, which was identified as a $P$. citrinum, but not clustered with the other $P$. citrinum sequences, $n^{\circ} 23$ which was identified as $P$. adametzii, and CBS 110769 , a P. nordicum reference strain. In COI dendrogram bootstrap values which support the clustering were far lower, but above $50 \%$.

Therefore, identification based on morphology was only partially confirmed: all the strains belonging to $P$. crustosum and $P$. citrinum were correctly identified. On the contrary, using the molecular approach, putative $P$. nalgiovense strains were identified as $P$. solitum.

The final molecular identification of the strains was obtained merging results from both gene sequencing and sequence clustering: 12 (44.4\%) P. crustosum (10 of which univocally identified by both of the 2 genes considered), 8 (29.6\%) P. solitum and 2 (7.4\%) P. citrinum strains were identified. Moreover, 3 other species ( $P$. brevicompactum, $P$. charlesii and $P$. oxalicum) were identified as single strains. After the final identification, all the strains were deposited in the MUM (Micoteca da Universidade do Minho, Braga, Portugal) culture collection and a code was assigned (Table 3 ).

\subsection{Mycotoxins}

Regarding mycotoxin contamination, OTA was detected in all the grana cheese samples, ranging from 1 to $1432 \mu \mathrm{g} / \mathrm{kg}$ whereas CIT was detected only in 4 cheese wheels (from storehouses $7,13,15,17$ ) ranging from 42 to $100 \mu \mathrm{g} / \mathrm{kg}$ (Table 2).

\section{Discussion}

Morphological observation of the colonies on CZ, CYA and MEA, was useful to group strains and get a first presumptive identification: $P$. crustosum strains looked very similar to the reference strains CBS 115503 and CBS 101025 and their assignment to the correct species was quite straightforward. On the contrary, P. solitum strains were more difficult to assign, being quite similar in shape, size and general appearance to colonies of other species, including $P$. nalgiovense.

Thus, to get an accurate and reliable identification at species level the use of molecular techniques was crucial. Results obtained from the sequencing of both genes were generally in agreement, but BenA region seems more suitable, having shown a very good clustering, both for the strains obtained from cheese and for the reference strains.
Only a few outliers were found. Strain $n^{\circ} 20$ was identified as $P$. corylophilum, but since it was far from the $P$. corylophilum sequences deposited in the GenBank and since its identity was not confirmed in COI sequencing, identification remained uncertain. Strain $n^{\circ} 22$ was identified as P. jugoslavicum in BenA dendrogram, but no reference sequence was available in the GenBank for COI region; therefore, in this case, it was identified as the closely related species $P$. adametzioides (Visagie et al., 2013). Strain $n^{\circ} 23$, based on BenA, was identified as P. charlesii, $P$. adametzii according to $\mathrm{COI}$ sequencing; no $P$. charlesii sequence was available for COI gene.

Strains $n^{\circ} 6$ and 18 showed no clear evidence of being P. crustosum from a molecular point of view, since it was not possible to amplify their BenA regions and they clustered separately in the COI dendrogram; therefore, molecular and morphological identifications are in disagreement.

Penicillium brevicompactum, $P$. crustosum, $P$. citrinum and $P$. solitum were reported in past studies in cheese and/or cheese manufacturing and ripening environments (Houbraken et al., 2010; Kure and Skaar, 2000; Kure et al., 2004; Samson and Frisvad, 2004; Serra et al., 2003), as well as $P$. citrinum and $P$. crustosum, but none of them is known as OTA producer.

OTA and CIT were the only mycotoxins considered in this study, because OTA has been reported in grated cheese (Biancardi et al., 2013) and the main possible producers were $P$. nordicum (OTA producer) and $P$. verrucosum (CIT and OTA producer). OTA was detected in all the samples, ranging from 1 to $1431.9 \mu \mathrm{g} / \mathrm{kg}$, but none of the producing species was detected using the classic, media-dependent isolation approach and the molecular characterisation of selected strains. Probably, presence of OTA producing species was limited compared with $P$. crustosum and P. solitum and this contributed to the difficulty of their isolation/selection. On the other hand, CIT presence, previously reported in cheese by Taniwaki and van Dender (2001) is supported by the isolation of $P$. citrinum. In the present study, the average OTA concentration was $183 \mu \mathrm{g} / \mathrm{kg}$. Contamination detected was commonly higher than that reported by Biancardi et al. (2013), between 1.62 and $54.07 \mu \mathrm{g} /$ $\mathrm{kg}$. This is not surprising because the data are not exactly comparable. Biancardi et al. (2013) analysed commercially packed grated cheese where $18 \% \mathrm{v} / \mathrm{v}$ of cheese rind is allowed, whereas rind constituted $100 \%$ in our samples. OTA presence was also reported in semi-hard cheese by Pattono et al. (2013) in concentrations ranging from 1.0 to $262.2 \mu \mathrm{g} / \mathrm{kg}$, in 6 of 32 cheese rind samples. Rind surface contamination during ripening is supposed to be responsible for OTA contamination;

Table 2

Fungal bioload, including Penicillium spp., and CIT and OTA concentrations obtained in the eighteen grana cheese samples of ripening storehouses studied.

\begin{tabular}{llllll}
\hline $\begin{array}{l}\text { Storehouse } \\
\text { code }\end{array}$ & $\begin{array}{l}\text { Storehouse } \\
\text { place }\end{array}$ & $\begin{array}{l}\text { Fungi } \\
\text { CFU/g }\end{array}$ & $\begin{array}{l}\text { Penicillium } \\
\text { CFU/g }\end{array}$ & $\begin{array}{l}\text { CIT } \\
\mu \mathrm{g} / \mathrm{kg}\end{array}$ & $\begin{array}{l}\text { OTA } \\
\mu \mathrm{g} / \mathrm{kg}\end{array}$ \\
\hline 1 & BS & $1 * 10^{7}$ & $4 * 10^{6}$ & $<$ LOD & 145 \\
2 & BS & $4 * 10^{5}$ & $8 * 10^{4}$ & $<$ LOD & 5 \\
3 & BS & $6 * 10^{3}$ & $3 * 10^{2}$ & $<$ LOD & 2 \\
4 & BS & $1 * 10^{5}$ & $1 * 10^{4}$ & $<$ LOD & 1 \\
5 & BS & $6 * 10^{4}$ & $4 * 10^{2}$ & $<$ LOD & 1432 \\
6 & BS & $7 * 10^{3}$ & $1 * 10^{2}$ & $<$ LOD & 126 \\
7 & BS & $7 * 10^{6}$ & $3 * 10^{5}$ & 97 & 96 \\
8 & CR & $1 * 10^{4}$ & $1 * 10^{2}$ & $<$ LOD & 17 \\
9 & CR & $8 * 10^{3}$ & $2 * 10^{3}$ & $<$ LOD & 3 \\
10 & CR & $4 * 10^{6}$ & $5 * 10^{5}$ & $<$ LOD & 221 \\
11 & CR & $3 * 10^{6}$ & $2 * 10^{5}$ & $<$ LOD & 6 \\
12 & MN & $6 * 10^{6}$ & $3 * 10^{4}$ & $<$ LOD & 543 \\
13 & MN & $2 * 10^{7}$ & $1 * 10^{5}$ & 100 & 77 \\
14 & MN & $5 * 10^{3}$ & $3 * 10^{2}$ & $<$ LOD & 153 \\
15 & MN & $7 * 10^{7}$ & No & 42 & 8 \\
16 & PC & $7 * 10^{7}$ & $1 * 10^{6}$ & $<$ LOD & 8 \\
17 & PC & $3 * 10^{6}$ & No & 68 & 401 \\
18 & VR & $3 * 10^{6}$ & No & $<$ LOD & 51 \\
\hline
\end{tabular}

LOD: limit of detection $=0.5 \mu \mathrm{g} / \mathrm{kg}(\mathrm{CIT})$ and $0.1 \mu \mathrm{g} / \mathrm{kg}$ (OTA). 
Table 3

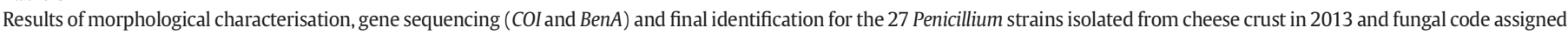
in the MUM culture collection.

\begin{tabular}{|c|c|c|c|c|c|c|}
\hline Strain & Store house & Morph. ID & $\mathrm{COI}$ & BenA & Final ID & MUM code \\
\hline 1 & 10 & P. citrinum & P. citrinum & P. citrinum & P. citrinum & 16.09 \\
\hline 2 & 12 & P. citrinum & P. citrinum & P. citrinum & P. citrinum & 16.10 \\
\hline 3 & 9 & P. crustosum & P. crustosum & P. crustosum & P. crustosum & 16.11 \\
\hline 4 & 16 & P. crustosum & P. crustosum & P. crustosum & P. crustosum & 16.120 \\
\hline 5 & 17 & P. crustosum & P. crustosum & P. crustosum & P. crustosum & 16.12 \\
\hline 6 & 17 & P. crustosum & P. crustosum & - & P. crustosum & 16.121 \\
\hline 7 & 7 & P. crustosum & P. crustosum & P. crustosum & P. crustosum & 16.122 \\
\hline 8 & 7 & P. crustosum & P. crustosum & P. crustosum & P. crustosum & 16.123 \\
\hline 9 & 7 & P. nalgiovense & P. solitum & P. solitum & P. solitum & 16.13 \\
\hline 10 & 15 & P. nalgiovense & P. solitum & P. solitum & P. solitum & 16.14 \\
\hline 11 & 16 & P. crustosum & P. crustosum & P. crustosum & P. crustosum & 16.124 \\
\hline 12 & 12 & P. crustosum & P. crustosum & P. crustosum & P. crustosum & 16.125 \\
\hline 13 & 17 & P. crustosum & P. crustosum & P. crustosum & P. crustosum & 16.126 \\
\hline 14 & 4 & P. nalgiovense & P. solitum & P. solitum & P. solitum & 16.127 \\
\hline 15 & 10 & P. nalgiovense & P. solitum & P. solitum & P. solitum & 16.128 \\
\hline 16 & 18 & P. crustosum & P. crustosum & P. crustosum & P. crustosum & 16.129 \\
\hline 17 & 9 & P. crustosum & P. crustosum & - & P. crustosum & 16.130 \\
\hline 18 & 16 & Penicillium sp. & P. oxalicum & - & P. oxalicum & 16.131 \\
\hline 19 & 9 & P. crustosum & P. crustosum & P. crustosum & P. crustosum & 16.132 \\
\hline 20 & 8 & Penicillium sp. & P. citrinum & P. corylophilum & Uncertain & 16.133 \\
\hline 21 & 8 & P. nalgiovense & P. solitum & P. solitum & P. solitum & 16.135 \\
\hline 22 & 17 & Penicillium sp. & P. adametzioides & P. jugoslavicum & Uncertain & 16.136 \\
\hline 23 & 17 & Penicillium sp. & P. adametzii & P. charlesii & P. charlesii & 16.137 \\
\hline 24 & 17 & P. brevicompactum & P. brevicompactum & P. brevicompactum & P. brevicompactum & 16.15 \\
\hline 25 & 8 & P. nalgiovense & P. solitum & P. solitum & P. solitum & 16.138 \\
\hline 26 & 8 & P. nalgiovense & P. solitum & P. solitum & P. solitum & 16.139 \\
\hline 27 & 16 & P. nalgiovense & P. solitum & P. solitum & P. solitum & 16.140 \\
\hline
\end{tabular}

-: no sequence available.

therefore, data from literature are reasonably in agreement with those reported in this study.

CIT in cheese was reported by Jarvis (1983) who investigated 44 cheese samples and found this mycotoxin in 17 of them (39\%) in concentrations up to $50 \mu \mathrm{g} / \mathrm{kg}$; thus, also these data are comparable with our results, with $22 \%$ of samples positive and contamination between 42 and $97 \mu \mathrm{g} / \mathrm{kg}$.

CIT, penitrem A and roquefortine C (Samson and Frisvad, 2004) could be detected in cheese, based on P. citrinum and P. crustosum fungi isolation in this study, in agreement with literature (Kokkonen et al., 2005; Richard and Arp, 1979). It is not clear which fungus is responsible for OTA contamination, because of the lack of isolation of the candidates $P$. verrucosum and $P$. nordicum. Therefore, this must be considered in future studies.

\section{Conclusions}

This work has provided for the first time information about Penicillia growing on grana cheese crust during ripening. Two dominant species, $P$. crustosum and $P$. solitum, emerged clearly from this survey, but neither is reported as an OTA-producer. Thus, the origin of OTA in all the cheese crust samples remains unknown. Ochratoxigenic species, although present to a lesser extent than the dominant fungi, must be considered and detecting methods other than the classic media-dependent isolation approach should be considered: since the latter is time-consuming and sometimes unreliable, support of molecular approaches is mandatory.

In conclusion, further studies on a broader set of strains are required to corroborate these data, contribute to better identification of the mycobiota and provide input for effective strategies to mitigate mycotoxin contamination.

\section{Acknowledgements}

The present work was supported by the following institutions: Consorzio del Formaggio Parmigiano Reggiano, Consorzio per la tutela del Formaggio Grana Padano, Fondazione Romeo ed Enrica Invernizzi.
The authors thank Prof. Terenzio Bertuzzi (Food Science and Nutrition Institute, Università Cattolica del Sacro Cuore, Piacenza, Italy) for mycotoxins analysis and Dr. Célia Soares (Centre of Biological Engineering, Universidade do Minho, Braga, Portugal) for preservation and depositing working at MUM.

\section{References}

Alzohairy, A.M., 2011. BioEdit: an important software for molecular biology. GERF B. B. 2, 60-61.

Ambrose, A.M., Deeds, F., 1946. Some toxicological and pharmacological properties of citrinin. J. Pharmacol. Exp. Ther. 88, 173-186.

Bailly, J.D., Querin, A., Le Bars-Bailly, S., Benard, G., Guerre, P., 2002. Citrinin production and stability in cheese. J. Food Prot. 65, 1317-1321.

Biancardi, A., Piro, R., Galaverna, G., Dall'Asta, C., 2013. A simple and reliable liquid chromatography-tandem mass spectrometry method for determination of ochratoxin A in hard cheese. Int. J. Food Sci. Nutr. 64, 632-640.

Cooper, S.J., Wood, G.M., Chapman, W.B., Williams, A.P., 1982. Mycotoxins occurring in mould damaged foods. Vth International IUPAC Symposium on Mycotoxins and Phycotoxins, Vienna, 1-3 September, pp. 64-67.

Dall'Asta, C., De Dea Lindner, J., Galaverna, G., Dossena, A., Neviani, E., Marchelli, R., 2008. The occurrence of ochratoxin A in blue cheese. Food Chem. 106, 729-734

Dragoni, I., Cantoni, C., Corti, S., 1983. Inquinamento fungino di Grana Padano da specie tossigene. Il Lattante 8, 605-607.

Felsenstein, J., 1985. Confidence limits on phylogenies: an approach using the bootstrap. Evolution 39, 783-791

Glass, N.L., Donaldson, G.C., 1995. Development of premier sets designed for use with the PCR to amplify conserved genes from filamentous Ascomycetes. Appl. Environ. Microbiol. 61, 1323-1330.

Houbraken, J., Frisvad, J.C., Samson, R.A., 2010. Taxonomy of Penicillium citrinum and related species. Fungal Divers. 44, 117-133.

IARC (International Agency for Research on Cancer), 1986. Some naturally occurring and synthetic food components, furocoumarins and ultraviolet radiation. IARC Monogr Eval. Carcinog. Risks Hum. 40 (444 pp).

IARC (International Agency for Research on Cancer), 1987. Overall evaluation of carcinogenicity: an updating to IARC monographs volumes 1 to 42. IARC Monogr. Eval. Carcinog. Risks Hum. Suppl. 7 (440 pp).

Jarvis, B., 1983. Mould and mycotoxins in mouldy cheese. Microbiologie-Aliments-Nutrition 1, 187-191.

Kimura, M., 1980. A simple method for estimating evolutionary rate of base substitutions through comparative studies of nucleotide sequences. J. Mol. Evol. 16, 111-120.

Kokkonen, M., Jestoi, M., Rizzo, A., 2005. Determination of selected mycotoxins in mould cheeses with liquid chromatography coupled to tandem with mass spectrometry. Food Addit. Contam. 22, 449-456. 
Kumar, S., Stecher, G., Tamura, K., 2016. MEGA7: molecular evolutionary genetics analysis version 7.0 for bigger datasets. Mol. Biol. Evol. http://dx.doi.org/10.1093/molbev/ msw054.

Kure, F.C., Skaar, I., 2000. Mould growth on the Norwegian semi-hard cheeses Norvegia and Jarlsberg. World Mycotoxin J. 62, 133-137.

Kure, F.C., Skaar, I., Brendehaug, J., 2004. Mould contamination in production of semi-hard cheese. Int. J. Food Microbiol. 93, 41-49.

Lund, F., Filtenborg, O., Frisvad, J.C., 1995. Associated mycoflora of cheese. Food Microbiol. $12,173-180$.

Lurá, M.C., Fuentes, M., Cabagna, M., González, A.M., Nepote, A., Giugni, M.C., Rico, M Latorre, M.G., 2004. Structural and ultrastructural alterations in BALB/c mice: effects of Penicillium citrinum metabolites. Mycopathologia 158, 233-238.

Montagna, M.T., Santacroce, M.P., Spilotros, G., Napoli, C., Minervini, F., Papa, A., Dragoni, I., 2004. Investigation of fungal contamination in sheep and goat cheeses in southern Italy. Mycopathologia 158, 245-249.

Ostry, V., Malir, F., Ruprich, J., 2013. Producers and important dietary sources of ochratoxin A and citrinin. Toxins 5, 1574-1586.

Pattono, D., Grosso, A., Stocco, P.P., Pazzi, M., Zeppa, G., 2013. Survey of the presence of patulin and ochratoxin A in traditional semi-hard cheeses. Food Control 33, 54-57.

Pitt, J.I., 1979. The Genus Penicillium and Its Teleomorphic States Eupenicillium and Talaromyces. Academic Press Inc. (634 pp)

Pugazhenthi, T.R., Dhanalakshmi, B., Narasimhan, R., Purushothaman, V, 2000. Isolation and toxicological screening of toxigenic Penicillium citrinum from cheese. Egypt J. Dairy Sci. 28, 163-168.

Richard, J.L., 2007. Some major mycotoxins and their mycotoxicoses-an overview. Int. J. Food Microbiol. 119, 3-10.
Richard, J.L., Arp, L.H., 1979. Natural occurrence of the mycotoxin penitrem A in moldy cream cheese. Mycopathologia 67, 107-109.

Ropars, J., Cruaud, C., Lacoste, S., Dupont, J., 2012. A taxonomic and ecological overwiew of cheese fungi. Int. J. Food Microbiol. 155, 199-210.

Saitou, N., Nei, M., 1987. The neighbor-joining method: a new method for reconstructing phylogenetic trees. Mol. Biol. Evol. 4, 406-425.

Samson, R.A., Frisvad, J.C., 2004. Penicillium subgenus Penicillium: new taxonomy schemes and mycotoxins and other extrolites. Stud. Mycol. 49, 1-266.

Seifert, K.A., Samson, R.A., de Waard, J.R., Houbraken, J., Levesque, C.A., Moncalvo, J.M., Louis-Seize, G., Hebert, P.D.N., 2007. Prospects for fungus identification using CO1 DNA barcodes, with Penicillium as a test case. Proc. Natl. Acad. Sci. U. S. A. 104, 3901-3906.

Sengun, I.Y., Yaman, D.B., Gonul, S.A., 2008. Mycotoxins and mould contamination in cheese: a review. World Mycotoxin J. 1, 291-298.

Serra, R., Abrunhosa, L., Kozakiewicz, Z., Venâncio, A., Lima, N., 2003. Use of ozone to reduce molds in a cheese ripening room. J. Food Prot. 66, 2355-2358.

Somorin, Y., Akinyemi, A., Bertuzzi, T., Pietri, A., 2016. Co-occurrence of aflatoxins, ochratoxin A and citrinin in "egusi" melon (Colocynthis citrullus L.) seeds consumed in Ireland and the United Kingdom. Food Addit. Contam., Part B 9, 230-235.

Taniwaki, M.H., van Dender, A.G.F., 2001. Growth of fungi and mycotoxin production on cheese under modified atmospheres. Int. J. Food Microbiol. 68, 125-133.

Visagie, C.M., Houbraken, J., Rodriques, C., Silva Pereira, C., Dijksterhuis, J., Seifert, K.A., Jacobs, K., Samson, R.A., 2013. Five new Penicillium species in section Sclerotiora: a tribute to the Dutch royal family. Persoonia 31, 42-62. 